\title{
Stability of time-varying systems in the absence of strict Lyapunov functions
}

\author{
Mohammad Fuad Mohammad Naser ${ }^{(1)}$; Fayçal Ikhouane $^{(2)}$ \\ (1) Faculty of Engineering Technology, Al-Balqa Applied University, \\ Amman 11134, Jordan. E-mail: mohammad.naser@bau.edu.jo \\ (2) Universitat Politècnica de Catalunya, Department of Mathematics, \\ Barcelona East School of Engineering, carrer Eduard Maristany, \\ 16, 08019, Barcelona, Spain. E-mail: faycal.ikhouane@upc.edu
}

\begin{abstract}
When a nonlinear system has a strict Lyapunov function, its stability can be studied using standard tools from Lyapunov stability theory. What happens when the strict condition fails? This paper provides an answer to that question using a formulation that does not make use of the specific structure of the system model. This formulation is then applied to the study of the asymptotic stability of some classes of linear and nonlinear time-varying systems. Lyapunov functions, time-varying systems, (asymptotic) stability.
\end{abstract}

\section{Introduction}

This paper deals with the asymptotic stability of the nonlinear time-varying system $\dot{x}=$ $f(t, x)$. When this system has a continuously differentiable Lyapunov function $V$ that is strict, standard results from Lyapunov stability theory establish the asymptotic stability of the origin [2, Theorem 3.2]. The strict condition on the Lyapunov function means that there exists a $\mathscr{K}$-function $\beta: \mathbb{R}_{+} \rightarrow \mathbb{R}_{+}$(that is $\beta$ is continuous and strictly increasing with $\beta(0)=0)$ that satisfies $\dot{V}(t, x) \leq-\beta(|x|)$.

What happens when the strict condition does not hold? The following sufficient conditions imply asymptotic stability in the absence of the strict condition on $V$.

(i) The origin is asymptotically stable under the condition that there exists $T>0$ such that for all $t_{0}$, and $x_{0}: V\left(t_{0}+T, x\left(t_{0}+T\right)\right)-V\left(t_{0}, x_{0}\right) \leq-\beta\left(\left|x_{0}\right|\right)([1])$. 
(ii) A strict Lyapunov function can be constructed if we know a Lyapunov function that satisfies $\dot{V}(t, x) \leq-W(q(t), x)$ for some periodic function $q$ and a function $W$ positive definite in $x$ (see [7]). The periodicity condition is weakened in [6].

(iii) The origin is asymptotically stable if $\dot{V}(t, x) \leq-\beta(|x|)+\gamma\left(t-t_{0}\right)$ for some $\mathscr{L}-$ function $\gamma: \mathbb{R} \rightarrow \mathbb{R}_{+}$; that is $\gamma$ is continuous, nonnegative, nonincreasing and $\lim _{t \rightarrow \infty} \gamma(t)=0$ (see 8 ).

(iv) Using the nested-Matrosov's theorem, it can be shown that the origin is uniformly globally asymptotically stable if $\dot{V}(t, x) \leq-\beta(|f(t, x)|)$ where all time derivatives of $f$ are bounded [5, Corollary 2].

What happens when the Lyapunov function is not strict, and the aforementioned sufficient conditions do not hold? The initial motivation of the present paper was to propose an answer to that question as follows: the origin is asymptotically stable if $\dot{V}(t, x) \leq-h(t)|x|^{b}$ for some nonnegative function $h$ such that $\int_{0}^{\infty} h(t) d t=\infty$. However, in the process of proving this fact, we realized that this result can be obtained from a more general formulation which be stated as follows: given a nonnegative absolutely continuous function $z$ whose explicit expression is not known, provide sufficient conditions on $z$ so that (1) we have an upper bound on $z$, and (2) we have $\lim _{t \rightarrow \infty} z(t)=0$.

This formulation is given in Section 3 as Propositions 2 and 3 . These two propositions are applied to the asymptotic stability of nonlinear time-varying systems in Section 4. The main result of Section 4 is Theorem 6 which provides sufficient conditions for the (global) asymptotic stability of equilibria. Another application to Propositions 2 and 3 is provided in Section 5 where we derive a new result on the asymptotic stability of perturbed linear systems in Theorem 10. The main novelty of the result is that the perturbations are allowed to be unbounded with respect to time. Conclusions are presented in Section 6.

\section{Terminology and notations}

A real number $x$ is said to be strictly positive when $x>0$, strictly negative when $x<0$, nonpositive when $x \leq 0$, and nonnegative when $x \geq 0$. A function $h: \mathbb{R} \rightarrow \mathbb{R}$ is said to be strictly increasing when $t_{1}<t_{2} \Rightarrow h\left(t_{1}\right)<h\left(t_{2}\right)$, strictly decreasing when $t_{1}<t_{2} \Rightarrow h\left(t_{1}\right)>h\left(t_{2}\right)$, nonincreasing when $t_{1}<t_{2} \Rightarrow h\left(t_{1}\right) \geq h\left(t_{2}\right)$, and nondecreasing when $t_{1}<t_{2} \Rightarrow h\left(t_{1}\right) \leq h\left(t_{2}\right)$. 11 The set of nonnegative integers is denoted $\mathbb{N}=\{0,1, \ldots\}$

\footnotetext{
${ }^{1}$ In this paper we avoid the use of the words "positive", "negative", "increasing", "decreasing" as they mean different things in different books.
} 
and the set of nonnegative real numbers is denoted $\mathbb{R}_{+}=[0, \infty)$. The Lebesgue measure on $\mathbb{R}$ is denoted $\mu$. We say that a subset of $\mathbb{R}$ is measurable when it is Lebesgue measurable. Let $I \subset \mathbb{R}_{+}$be an interval, and consider a function $p: I \rightarrow \mathbb{R}^{l}$ where $l>0$ is an integer. We say that $p$ is measurable when $p$ is $\left(M_{\mu}, B\right)$-measurable where $B$ is the class of Borel sets of $\mathbb{R}^{l}$ and $M_{\mu}$ is the class of measurable sets of $\mathbb{R}_{+}$(see [13]). For a measurable function $p: I \rightarrow \mathbb{R}^{l},\|p\|_{I}$ denotes the essential supremum of the function $|p|$ on $I$ where $|\cdot|$ is the Euclidean norm on $\mathbb{R}^{l}$. When $I=\mathbb{R}_{+}$, this essential supremum is denoted $\|p\| . C^{0}\left(\mathbb{R}, \mathbb{R}^{n}\right)$ denotes the Banach space of continuous functions $u: \mathbb{R} \rightarrow \mathbb{R}^{n}$ endowed with the uniform convergence norm $\|\cdot\|$. For each open interval $I \subset \mathbb{R}$, the set of all continuously differentiable functions $u: I \rightarrow \mathbb{R}^{n}$ is denoted $C^{1}\left(I, \mathbb{R}^{n}\right)$.

\section{Stability results}

The main results of this section are Propositions 2 and 3 which provide an answer to the following question: consider an absolutely continuous function $z \geq 0$; if we do not have the explicit expression of $z$, what kind of knowledge do we need in order to (1) establish an upper bound on $z$, and (2) show that $\lim _{t \rightarrow \infty} z(t)=0$ ? The sufficient conditions provided in Propositions 2 and 3 generalise the following lemma from [9].

Lemma 1. [9, Lemma 17] Consider a function $z:\left[t_{0}, \omega\right) \rightarrow \mathbb{R}_{+}$where $t_{0} \in \mathbb{R}$ and $t_{0}<\omega \leq \infty$. Assume that Conditions (i)-(ii) hold.

(i) The function $z$ is absolutely continuous on each compact interval of $\left[t_{0}, \omega\right)$.

(ii) There exist $z_{1} \geq 0$ and $z_{2}>0$ such that $z_{1}<z_{2}, z\left(t_{0}\right)<z_{2}$ and $\dot{z}(t) \leq 0$ for almost all $t \in\left(t_{0}, \omega\right)$ that satisfy $z_{1}<z(t)<z_{2}$.

Then $z(t) \leq \max \left(z\left(t_{0}\right), z_{1}\right), \forall t \in\left[t_{0}, \omega\right)$.

Proposition 2. Consider a function $z:\left[t_{0}, \omega\right) \rightarrow \mathbb{R}_{+}$where $t_{0} \in \mathbb{R}$ and $t_{0}<\omega \leq \infty$. Assume that Conditions (i)-(iii) hold.

(i) The function $z$ is absolutely continuous on each compact interval of $\left[t_{0}, \omega\right)$.

(ii) There exist functions $\varphi, \psi:\left[t_{0}, \omega\right) \rightarrow \mathbb{R}_{+}$such that

(ii) -1 both $\varphi$ and $\psi$ are absolutely continuous on each compact interval of $\left[t_{0}, \omega\right)$,

(ii)-2 and Inequalities (9)-10) hold,

$$
\begin{gathered}
\varphi(t)<\psi(t), \forall t \in\left[t_{0}, \omega\right), \\
\dot{z}(t) \leq \min (\dot{\varphi}(t), \dot{\psi}(t)), \text { for almost all } t \in\left(t_{0}, \omega\right) \\
\text { that satisfy } \varphi(t)<z(t)<\psi(t) .
\end{gathered}
$$


(iii) $z\left(t_{0}\right) \leq \varphi\left(t_{0}\right)$.

Then

$$
z(t) \leq \varphi(t), \forall t \in\left[t_{0}, \omega\right) .
$$

Furthermore, if $\varphi$ is nonincreasing and

$$
\dot{z}(t) \leq 0, \text { for almost all } t \in\left(t_{0}, \omega\right) \text { that satisfy } z(t)<\varphi(t)
$$

then

$$
z(t) \leq \min \left(z\left(t_{0}\right), \varphi(t)\right), \forall t \in\left[t_{0}, \omega\right) .
$$

Proof. The aim of the following analysis is to prove that Inequality (3) holds. To this end, we use an argument by contradiction. Assume that there exists some $t_{1} \in\left(t_{0}, \omega\right)$ such that $z\left(t_{1}\right)>\varphi\left(t_{1}\right)$. Consider the set $C=\left\{\tau \in\left[t_{0}, \omega\right) / z(t) \leq \varphi(t)\right.$, for all $\left.t \in\left[t_{0}, \tau\right]\right\}$. The set $C$ is nonempty because $t_{0} \in C$. Define $t_{2}=\sup C$. Observe that $t_{2} \leq t_{1}<\infty$ because otherwise we have $t_{1} \in C$ which contradicts the fact that $z\left(t_{1}\right)>\varphi\left(t_{1}\right)$. By the definition of $t_{2}$, there exists a real sequence $\left\{\tau_{n} \in C\right\}_{n=1}^{\infty}$ such that $\lim _{n \rightarrow \infty} \tau_{n}=t_{2}$. The continuity of functions $z$ and $\varphi$ implies that $z\left(t_{2}\right)=\lim _{n \rightarrow \infty} z\left(\tau_{n}\right) \leq \lim _{n \rightarrow \infty} \varphi\left(\tau_{n}\right)=\varphi\left(t_{2}\right)$. This fact implies that $t_{2} \in C$ leading to $t_{2}<t_{1}$. Also, there exists a real sequence $\left\{\tau_{n}^{\prime}>t_{2}\right\}_{n=1}^{\infty}$ such that $z\left(\tau_{n}^{\prime}\right)>\varphi\left(\tau_{n}^{\prime}\right)$, for all $n \in \mathbb{N}$ and $\lim _{n \rightarrow \infty} \tau_{n}^{\prime}=t_{2}$. Since $z$ and $\varphi$ are continuous we get $z\left(t_{2}\right) \geq \varphi\left(t_{2}\right)$ which leads to $z\left(t_{2}\right)=\varphi\left(t_{2}\right)$.

Let $D=\left\{t \in\left[t_{2}, t_{1}\right] / z(t)=\varphi(t)\right\}$. The set $D$ is nonempty because $t_{2} \in D$. Define $t_{3}=\sup D$, then using a similar argument as above we get $t_{3} \in D$ which implies that $z\left(t_{3}\right)=\varphi\left(t_{3}\right)$ and $t_{3}<t_{1}$.

Claim 1. For all $t_{3}<t \leq t_{1}, z(t)>\varphi(t)$.

Proof. To prove the claim we use an argument by contradiction. Assume that there exists $t_{4} \in\left(t_{3}, t_{1}\right]$ such that $z\left(t_{4}\right) \leq \varphi\left(t_{4}\right)$. By the definition of $t_{3}$, we get $z\left(t_{4}\right)<\varphi\left(t_{4}\right)$. Define the function $z_{1}:\left[t_{0}, \omega\right) \rightarrow \mathbb{R}$ as $z_{1}(t)=z(t)-\varphi(t)$, for all $t \in\left[t_{0}, \omega\right)$. The function $z_{1}$ is continuous and satisfies $z_{1}\left(t_{4}\right)<0$ and $z_{1}\left(t_{1}\right)>0$. Thus we conclude from the Intermediate Value Theorem that there exists some $t_{5} \in\left(t_{4}, t_{1}\right)$ such that $z_{1}\left(t_{5}\right)=0$ so that $z\left(t_{5}\right)=\varphi\left(t_{5}\right)$ which implies that $t_{5} \in D$. This is not possible since $t_{5}>t_{3}$. The proof of Claim 1 has been completed.

Let $E=\left\{t \in\left(t_{3}, t_{1}\right] / z(t)=\psi(t)\right\}$. Define

$$
t_{6}= \begin{cases}t_{1} & \text { if } E=\emptyset \\ \text { inf } E & \text { if } E \neq \emptyset\end{cases}
$$


Claim 2. $z\left(t_{6}\right)>\varphi\left(t_{6}\right)$.

Proof. If $E=\emptyset$, then $t_{6}=t_{1}$ and thus $z\left(t_{6}\right)>\varphi\left(t_{6}\right)$. If $E \neq \emptyset$, then $t_{6}=\inf E$ and thus the continuity of $z$ and $\psi$ implies that $t_{6} \in E$, so that $z\left(t_{6}\right)=\psi\left(t_{6}\right)$ and hence we get from (9) that $z\left(t_{6}\right)>\varphi\left(t_{6}\right)$. Thus, in all cases we have $z\left(t_{6}\right)>\varphi\left(t_{6}\right)$.

Claim 3. $z(t)<\psi(t)$, for all $t_{3}<t<t_{6}$.

Proof. If $E=\emptyset$, then $t_{6}=t_{1}$. We need to show that $z(t)<\psi(t)$, for all $t_{3}<t<t_{6}$. To this end, assume that there exists $t_{6}^{\prime} \in\left(t_{3}, t_{6}\right)$ such that $z\left(t_{6}^{\prime}\right) \geq \psi\left(t_{6}^{\prime}\right)$. Since $E=\emptyset$, we have $z\left(t_{6}^{\prime}\right)>\psi\left(t_{6}^{\prime}\right)$. Thus we deduce by Inequality (9) and the fact $z\left(t_{3}\right)=\varphi\left(t_{3}\right)$ that

$$
z_{1}\left(t_{6}^{\prime}\right)>0=z\left(t_{3}\right)-\varphi\left(t_{3}\right)>z\left(t_{3}\right)-\psi\left(t_{3}\right)=z_{1}\left(t_{3}\right),
$$

where $z_{1}(\cdot)=z(\cdot)-\psi(\cdot)$. Therefore, by applying the Intermediate Value Theorem on the continuous function $z_{1}(\cdot)$ and on the interval $\left[t_{3}, t_{6}^{\prime}\right]$, we deduce that $E \neq \emptyset$ which is a contradiction. This proves that $z(t)<\psi(t)$, for all $t_{3}<t<t_{6}$.

If $E \neq \emptyset$, it can be shown by the continuity of $z$ and $\psi$ that $t_{6}=\inf E \in E$ so that $z\left(t_{6}\right)=\psi\left(t_{6}\right)$. We need to show that $z(t)<\psi(t)$, for all $t_{3}<t<t_{6}$. To this end, assume that there exists $t_{6}^{\prime \prime} \in\left(t_{3}, t_{6}\right)$ such that $z\left(t_{6}^{\prime \prime}\right) \geq \psi\left(t_{6}^{\prime \prime}\right)$. Since $t_{6}=\inf E$, we have $z\left(t_{6}^{\prime \prime}\right)>\psi\left(t_{6}^{\prime \prime}\right)$. Thus we deduce by Inequality (9) and the fact $z\left(t_{3}\right)=\varphi\left(t_{3}\right)$ that

$$
z_{1}\left(t_{6}^{\prime \prime}\right)>0=z\left(t_{3}\right)-\varphi\left(t_{3}\right)>z\left(t_{3}\right)-\psi\left(t_{3}\right)=z_{1}\left(t_{3}\right),
$$

where $z_{1}(\cdot)=z(\cdot)-\psi(\cdot)$. Therefore, by applying the Intermediate Value Theorem on the continuous function $z_{1}(\cdot)$ and on the interval $\left[t_{3}, t_{6}^{\prime \prime}\right]$, we deduce that there exists $t_{6}^{\prime \prime \prime} \in\left(t_{3}, t_{6}^{\prime \prime}\right)$ such that $z_{1}\left(t_{6}^{\prime \prime \prime}\right)=0$ so that $z\left(t_{6}^{\prime \prime \prime}\right)=\psi\left(t_{6}^{\prime \prime \prime}\right)$. This contradicts the facts that $t_{6}=\inf E$ and $t_{3}<t_{6}^{\prime \prime \prime}<t_{6}^{\prime \prime}<t_{6}$ and thus $z(t)<\psi(t)$, for all $t_{3}<t<t_{6}$. This end the proof of Claim 3.

Since $t_{3}<t_{6} \leq t_{1}$, we get from Claims 1 and 3 that

$$
\varphi(t)<z(t)<\psi(t), \forall t_{3}<t<t_{6} .
$$

Thus we obtain by (10) that $\dot{z}(t) \leq \dot{\varphi}(t)$, for almost all $t \in\left(t_{3}, t_{6}\right)$. Hence the absolute continuity of functions $z$ and $\varphi$ leads to $z\left(t_{6}\right)-z\left(t_{3}\right)=\int_{t_{3}}^{t_{6}} \dot{z}(\tau) d \tau \leq \int_{t_{3}}^{t_{6}} \dot{\varphi}(\tau) d \tau=$ $\varphi\left(t_{6}\right)-\varphi\left(t_{3}\right)$ and thus we obtain by the fact $z\left(t_{3}\right)=\varphi\left(t_{3}\right)$ that $z\left(t_{6}\right) \leq \varphi\left(t_{6}\right)$ which contradicts Claim 2.

As a summary, we have proved that

$$
z(t) \leq \varphi(t), \forall t \in\left[t_{0}, \omega\right),
$$


which proves (3).

We need to prove that Inequality (5) is true whenever (4) is satisfied and $\varphi$ is nonincreasing. To this end, we prove that $z(t) \leq z\left(t_{0}\right)$, for all $t \in\left[t_{0}, \omega\right)$ for both situations $z\left(t_{0}\right)=\varphi\left(t_{0}\right)$ and $z\left(t_{0}\right)<\varphi\left(t_{0}\right)$.

If $z\left(t_{0}\right)=\varphi\left(t_{0}\right)$, then since $\varphi$ is nonincreasing, we obtain from (7) that

$$
z(t) \leq \varphi(t) \leq \varphi\left(t_{0}\right)=z\left(t_{0}\right), \text { for all } t \in\left[t_{0}, \omega\right),
$$

which means that (5) is satisfied.

If $z\left(t_{0}\right)<\varphi\left(t_{0}\right)$, suppose that there exists $t_{7} \in\left(t_{0}, \omega\right)$ such that $z\left(t_{7}\right)>z\left(t_{0}\right)$. Then $\varphi\left(t_{0}\right)>0$ (because if $\varphi\left(t_{0}\right)=0$, then $z\left(t_{0}\right)<0$ which is a contradiction). Let $S=$ $\left\{t \in\left(t_{0}, t_{7}\right] / z(t)=\varphi(t)\right\}$. We now show that $S$ cannot be empty. To this end, assume that $S=\emptyset$. Using (7) and the continuity of $z$ and $\varphi$, a simple contradiction argument proves that $z(t)<\varphi(t)$, for all $t \in\left[t_{0}, t_{7}\right)$. Hence we obtain from (4) that $z\left(t_{7}\right)-z\left(t_{0}\right)=$ $\int_{t_{0}}^{t_{7}} \dot{z}(\tau) d \tau \leq 0$ so that $z\left(t_{7}\right) \leq z\left(t_{0}\right)$. This contradicts the assumption $z\left(t_{7}\right)>z\left(t_{0}\right)$. Thus $S \neq \emptyset$. Let $t_{8}=\inf S$. Using (7) and the continuity of $z$ and $\varphi$, a simple contradiction argument proves that $t_{0}<t_{8} \leq t_{7}$ and $z(t)<\varphi(t)$, for all $t \in\left[t_{0}, t_{8}\right)$. Hence we obtain from (4) that $z\left(t_{8}\right)-z\left(t_{0}\right)=\int_{t_{0}}^{t_{8}} \dot{z}(\tau) d \tau \leq 0$ so that

$$
z\left(t_{8}\right) \leq z\left(t_{0}\right)
$$

The continuity of the functions $z$ and $\varphi$ implies that $t_{8}=\inf S \in S$ so that $z\left(t_{8}\right)=\varphi\left(t_{8}\right)$. Therefore, since $\varphi$ is nonincreasing and $t_{0}<t_{8} \leq t_{7}$, we get from (7) that

$$
z\left(t_{8}\right)=\varphi\left(t_{8}\right) \geq \varphi\left(t_{7}\right) \geq z\left(t_{7}\right)>z\left(t_{0}\right),
$$

which contradicts (8). Thus, Inequality (5) is satisfied and the proof has been completed.

Proposition 3. Consider a function $z:\left[t_{0}, \omega\right) \rightarrow \mathbb{R}_{+}$where $t_{0} \in \mathbb{R}$ and $t_{0}<\omega \leq \infty$. Assume that Conditions (i)-(iii) hold.

(i) The function $z$ is absolutely continuous on each compact interval of $\left[t_{0}, \omega\right)$.

(ii) There exist functions $\varphi, \psi:\left[t_{0}, \omega\right) \rightarrow \mathbb{R}_{+}$such that

(ii) -1 both $\varphi$ and $\psi$ are absolutely continuous on each compact interval of $\left[t_{0}, \omega\right)$, 
(ii)-2 and Inequalities (9)-10) hold,

$$
\begin{aligned}
& \varphi(t)<\psi(t), \forall t \in\left[t_{0}, \omega\right) \\
& \dot{z}(t) \leq \min (\dot{\varphi}(t), \dot{\psi}(t)) \text {, for almost all } t \in\left(t_{0}, \omega\right) \\
& \text { that satisfy } \varphi(t)<z(t)<\psi(t) \text {. }
\end{aligned}
$$

(iii) $z\left(t_{0}\right) \in\left(\varphi\left(t_{0}\right), \psi\left(t_{0}\right)\right)$.

Then

$$
z(t)<\psi(t), \forall t \in\left[t_{0}, \omega\right) .
$$

Assume furthermore that $\omega=\infty$ and that there exist a measurable function $q:\left[t_{0}, \infty\right) \rightarrow$ $\mathbb{R}_{+}$and a nondecreasing measurable function $H: \mathbb{R}_{+} \rightarrow \mathbb{R}_{+}$such that Equations (12)-(14) hold.

$$
H(0)=0 \text { and } H(v)>0, \forall v>0,
$$

$$
\begin{aligned}
\dot{z}(t) \leq-q(t) H(z(t)) & \text { for almost all } t \in\left(t_{0}, \infty\right) \\
& \text { that satisfy } \varphi(t)<z(t)<\psi(t), \\
& \lim _{t \rightarrow \infty} \int_{t_{0}}^{t} q(\tau) d \tau=\infty .
\end{aligned}
$$

Then (a) and (b) hold.

(a) If $L=\lim _{t \rightarrow \infty} \varphi(t)=0$, we have $\lim _{t \rightarrow \infty} z(t)=0$.

(b) If $L=\lim _{t \rightarrow \infty} \varphi(t)$ does not exist, is infinite, or exists with $L>0$, then there exists $T \in\left(t_{0}, \omega\right)$ such that

$$
\begin{aligned}
\varphi(t)<z(t) & \leq z\left(t_{0}\right), \forall t \in\left[t_{0}, T\right), \\
z(T) & =\varphi(T), \\
z(t) & \leq \varphi(t), \forall t \in(T, \infty) .
\end{aligned}
$$


Proof. The aim of the following analysis is to prove Inequality (11). To this end, consider the set $B=\left\{t \in\left(t_{0}, \omega\right) / z(t)=\varphi(t)\right\}$.

If $B=\emptyset$, assume that there exists $t_{1} \in\left(t_{0}, \omega\right)$ with $z\left(t_{1}\right) \geq \psi\left(t_{1}\right)$. Let $J=\left\{t \in\left(t_{0}, \omega\right) / z(t)=\psi(t)\right\}$. Using Intermediate Value Theorem, it can be verified that $J \neq \emptyset$. Let $t_{2}=\inf J$. Then for all $t \in\left[t_{0}, t_{2}\right)$ we have $z(t)<\psi(t)$. One can use the Intermediate Value Theorem to prove that $\varphi(t)<z(t)$, for all $t \in\left[t_{0}, t_{2}\right)$. These facts along with 10 imply that for almost all $t \in\left(t_{0}, t_{2}\right)$ we have $\dot{z}(t) \leq \dot{\psi}(t)$. Since $z$ and $\psi$ are absolutely continuous, it follows that $z\left(t_{2}\right)-z\left(t_{0}\right)=\int_{t_{0}}^{t_{2}} \dot{z}(\tau) d \tau \leq \int_{t_{0}}^{t_{2}} \dot{\psi}(\tau) d \tau=\psi\left(t_{2}\right)-\psi\left(t_{0}\right)$. This contradicts the facts that $z\left(t_{2}\right)=\psi\left(t_{2}\right)$ and $\psi\left(t_{0}\right)>z\left(t_{0}\right)$ and thus

$$
z(t)<\psi(t), \forall t \in\left[t_{0}, \omega\right) \text { whenever } B=\emptyset .
$$

If $B \neq \emptyset$, then $t_{3}=\inf B \in B$ because of the continuity of $z$ and $\varphi$. Thus seeing $t_{3}$ as an initial time, and $\varphi\left(t_{3}\right)$ as an initial condition, it follows from Proposition 2 that for all $t \in\left[t_{3}, \omega\right)$ we have $z(t) \leq \varphi(t)$. Using the same analysis used for $B=\emptyset$, it can be shown that $z(t)<\psi(t)$ for all $t \in\left[t_{0}, t_{3}\right)$. We summarize the previous results as

$$
\left\{\begin{array}{ll}
z(t)<\psi(t) & \forall t \in\left[t_{0}, t_{3}\right) \\
z(t) \leq \varphi(t) & \forall t \in\left[t_{3}, \omega\right)
\end{array} \quad \text { whenever } B \neq \emptyset\right.
$$

and thus Inequality (9) implies that $z(t)<\psi(t)$ for all $t \in\left[t_{0}, \omega\right)$, whenever $B \neq \emptyset$.

We conclude that, in both situations $B=\emptyset$ and $B \neq \emptyset$, Inequality (11) is satisfied.

To complete the proof assume that $\omega=\infty$ and that there exist a measurable function $q:\left[t_{0}, \infty\right) \rightarrow \mathbb{R}_{+}$and a nondecreasing measurable function $H: \mathbb{R}_{+} \rightarrow \mathbb{R}_{+}$for which (12), (13) and (14) are satisfied. Let $A=\left\{t \in\left[t_{0}, \infty\right) / z(t)=\varphi(t)\right\}$.

Claim 1. If $A=\emptyset$, then $\lim _{t \rightarrow \infty} z(t)=\lim _{t \rightarrow \infty} \varphi(t)=0$.

Proof. Since $A=\emptyset$, Inequality (11) and a simple contradiction argument prove that

$$
\varphi(t)<z(t)<\psi(t), \forall t \in\left[t_{0}, \infty\right) .
$$

Thus, we obtain from (13) that $\dot{z}(t) \leq 0$ for almost all $t \in\left(t_{0}, \infty\right)$ because the functions $q$ and $H$ are nonnegative. Thus $z$ is nonincreasing on $\left[t_{0}, \infty\right)$ so that $\lim _{t \rightarrow \infty} z(t)=L_{*}$ exists with $z(t) \geq L_{*} \geq 0$ for all $t \in\left[t_{0}, \infty\right)$.

If $L_{*}>0$ then, from (16), (13) and for all $t \in\left[t_{0}, \infty\right), z(t) \geq L_{*}$, it follows that

$$
\dot{z}(t) \leq-\epsilon q(t), \text { for almost all } t \in\left(t_{0}, \infty\right),
$$


where $\epsilon=H\left(L_{*}\right)$ is strictly positive because of $(12)$. Thus, the Fundamental Theorem of Calculus implies that

$$
z(t)=z\left(t_{0}\right)+\int_{t_{0}}^{t} \dot{z}(\tau) d \tau \leq z\left(t_{0}\right)-\epsilon \int_{t_{0}}^{t} q(t), \forall t \in\left[t_{0}, \infty\right)
$$

which leads to $\lim _{t \rightarrow \infty} z(t)=-\infty$ because of $(14)$. This contradiction proves that $L_{*}=\lim _{t \rightarrow \infty} z(t)=0$ and thus we obtain from (16) that $\lim _{t \rightarrow \infty} \varphi(t)=0$. This finishes the proof of Claim 1.

Claim 2. If $A \neq \emptyset$, then there exists $T \in\left(t_{0}, \omega\right)$ such that 15$)$ is satisfied.

Proof. The continuity of $z$ and $\varphi$ implies that $T=\inf A \in A$ so that $z(T)=\varphi(T)$. It can be easily verified using (13) and (11) that $\varphi(t)<z(t) \leq z\left(t_{0}\right)$ for all $t \in\left[t_{0}, T\right)$. Now, seeing $T$ as an initial time and $\varphi(T)$ as an initial condition, we obtain from Proposition 2 that $z(t) \leq \varphi(t)$ for all $t \in(T, \infty)$. Therefore 15$)$ is satisfied.

If $\lim _{t \rightarrow \infty} \varphi(t)=0$, we deduce from Claims 1 and 2 that $\lim _{t \rightarrow \infty} z(t)=0$. Otherwise, we get from Claim 1 that $A \neq \emptyset$ and thus (15) is satisfied because of Claim 2. This completes the proof.

The following corollary provides sufficient conditions to get $\lim _{t \rightarrow \infty} z(t)=0$.

Corollary 4. Suppose that Conditions (a)-(e) hold.

(a) Conditions (i) and (ii) of Proposition 2 are satisfied,

(b) $\omega=\infty$,

(c) $\lim _{t \rightarrow \infty} \varphi(t)=0$,

(d) $z\left(t_{0}\right)<\psi\left(t_{0}\right)$,

(e) there exist a measurable function $q:\left[t_{0}, \infty\right) \rightarrow \mathbb{R}_{+}$and a nondecreasing measurable function $H: \mathbb{R}_{+} \rightarrow \mathbb{R}_{+}$for which Equations (12), (13) and (14) are satisfied.

Then $\lim _{t \rightarrow \infty} z(t)=0$.

Proof. The result follows by considering the cases $z\left(t_{0}\right) \leq \varphi\left(t_{0}\right)$ and $z\left(t_{0}\right) \in\left(\varphi\left(t_{0}\right), \psi\left(t_{0}\right)\right)$ along with Proposition 2 and 3 respectively.

Remark. In [10] an attractiveness result is obtained using a different formulation and with different assumptions. It roughly says that when the derivative of some function is less than the sum of a negative function of the state and a 'small' -in some sense- function 
of the state, we get some kind of asymptotic convergence of the state to zero. This result is not directly related to Propositions 2 and 3 because the right-hand side of Inequality (13) contains two separate parts: a term that depends on the state and another that depends on time.

\section{Asymptotic stability of a class of nonlinear time--varying systems}

The aim of this section is to study the asymptotic stability of system (17)-18

$$
\begin{aligned}
\dot{x}(t) & =f(t, x(t)), t \in \mathbb{R}_{+}, \\
x\left(t_{0}\right) & =x_{0},
\end{aligned}
$$

where $\left(t_{0}, x_{0}\right) \in \mathbb{R}_{+} \times \mathbb{R}^{m}$, state $x(t)$ take values in $\mathbb{R}^{m}$ for some strictly positive integer $m$, and function $f:\left[t_{0}, \infty\right) \times \mathbb{R}^{m} \rightarrow \mathbb{R}^{m}$ is well-defined. Section 4.1 presents a brief summary of the results provided in [2] regarding the existence of solutions of the differential equation (17)-(18). In Section 4.2 we present the stability definitions that are used throughout the paper. Section 4.3 gives sufficient conditions for the asymptotic stability of the system (17).

\subsection{Carathéodory solutions}

The following set of assumptions for (17)-(18) is considered in [2, Section 1.1].

$\left(\mathrm{A}_{1}\right)$ The function $f(t, x)$ is locally essentially bounded on $\mathbb{R}_{+} \times \mathbb{R}^{m}$,

$\left(\mathrm{A}_{2}\right)$ for each $x \in \mathbb{R}^{m}$, the function $t \mapsto f(t, x)$ is measurable,

$\left(\mathrm{A}_{3}\right)$ for almost all $t \in \mathbb{R}_{+}$, the function $x \mapsto f(t, x)$ is continuous.

A function $x$ is called a local Carathéodory solution of $(17)-(18)$ on the interval $I \subset \mathbb{R}_{+}$ if $t_{0} \in I$, the function $x$ is absolutely continuous on every compact subinterval of $I$ and satisfies

$$
\begin{aligned}
\dot{x}(t) & =f(t, x(t)), \text { for almost all } t \in I, \\
x\left(t_{0}\right) & =x_{0} .
\end{aligned}
$$

Carathéodory's Theorem states that if Assumptions $\left(\mathrm{A}_{1}\right)-\left(\mathrm{A}_{3}\right)$ hold then for each initial pair $\left(t_{0}, x_{0}\right) \in \mathbb{R}_{+} \times \mathbb{R}^{m}$, then there exists an interval $I \subset \mathbb{R}_{+}$with $t_{0} \in I$ such that a local Carathéodory solution (19)-(20) exists on $I$.

If the function $f$ is continuous on $\mathbb{R}_{+} \times \mathbb{R}^{m}$ then Assumptions $\left(\mathrm{A}_{1}\right)-\left(\mathrm{A}_{3}\right)$ are satisfied. By Peano's Theorem it follows that a classical solution $x$ of (17)-(18) exists on some interval $t_{0} \in I \subset \mathbb{R}_{+}$. The term "classical" means that $x$ is of class $C^{1}$ and

$$
\begin{aligned}
\dot{x}(t) & =f(t, x(t)), \forall t \in I, \\
x\left(t_{0}\right) & =x_{0} .
\end{aligned}
$$




\subsection{Stability definitions}

In this section and in the remaining sections we assume that Assumptions $\left(A_{1}\right)-\left(A_{3}\right)$ hold so that at least one local Carathéodory solution (19)-(20) exists on some interval $t_{0} \in I \subset \mathbb{R}_{+}$.

Definition 5. [2, p.79] The point $x=0$ of the system (17) is said to be

(i) an equilibrium point if $f(t, 0)=0$ for almost all $t \geq 0$,

(ii) locally attractive if for each $t_{0} \in \mathbb{R}_{+}$, there exists $c>0$ such that if $\left|x_{0}\right|<c$, then each solution $x$ of $(17)-(18)$ is continuable on $\left[t_{0}, \infty\right)$ with $\lim _{t \rightarrow \infty} x(t)=0$,

(iii) globally attractive if for all $t_{0} \in \mathbb{R}_{+}$and all $x_{0} \in \mathbb{R}^{m}$, each solution $x$ of $(17)-(18)$ is continuable on $\left[t_{0}, \infty\right)$ with $\lim _{t \rightarrow \infty} x(t)=0$,

(iv) stable if for any $t_{0} \in \mathbb{R}_{+}$and any $\varepsilon>0$, there is $c>0$ such that if $\left|x_{0}\right|<c$ then each solution $x$ of $(17)-(18)$ is continuable on $\left[t_{0}, \infty\right)$ and

$$
|x(t)|<\varepsilon, \forall t \geq t_{0},
$$

(v) uniformly stable if for any $\varepsilon>0$, there is $c>0$ such that for each $t_{0} \in \mathbb{R}_{+}$and each $\left|x_{0}\right|<c$, every solution $x$ of $(17)-(18)$ is continuable on $\left[t_{0}, \infty\right)$ and

$$
|x(t)|<\varepsilon, \forall t \geq t_{0}
$$

(vi) asymptotically stable if it is stable and locally attractive,

(vii) globally asymptotically stable if it is stable and globally attractive.

\subsection{Sufficient conditions for the asymptotic stability}

In this section we use Proposition 2 and 3 to study the asymptotic stability of the system (17)-18). The main result is given in Theorem 6 .

For any $t_{0} \in \mathbb{R}$ define the set

$$
\Omega_{2}\left(t_{0}\right)=\left\{h: \mathbb{R} \rightarrow \mathbb{R}_{+} / h \text { is Lebesgue measurable with } \int_{t_{0}}^{\infty} h(s) d s=\infty\right\} .
$$

Theorem 6. Consider the system (17)- (18) for which Assumptions $\left(\mathrm{A}_{1}\right)-\left(\mathrm{A}_{3}\right)$ are satisfied. Assume that Conditions (i)-(ii) hold. 
(i) The origin $x=0$ is an equilibrium point for the system (17).

(ii) There exists a function $h \in \Omega_{2}\left(t_{0}\right)$ such that for each solution $x$ of (17)-(18) with maximal interval of existence $\left[t_{0}, \omega\right)$ there exist positive constants $\delta, a, b, k_{1}, k_{2}$, and a function $V \in C^{1}\left(\mathbb{R} \times \mathbb{R}^{m}, \mathbb{R}_{+}\right)$, satisfying

$$
\begin{gathered}
k_{1}|\alpha|^{a} \leq V(t, \alpha) \leq k_{2}|\alpha|^{a}, \forall t \in\left[t_{0}, \omega\right), \forall \alpha \in \mathbb{R}^{m}, \\
\left.\frac{\partial V(t, \alpha)}{\partial t}\right|_{\alpha=x(t)}+\left.\frac{\partial V(t, \alpha)}{\partial \alpha}\right|_{\alpha=x(t)} \cdot f(t, x(t)) \leq-h(t)|x(t)|^{b},
\end{gathered}
$$

for almost all $t \in\left(t_{0}, \omega\right)$ that satisfy $|x(t)|<\delta$.

Then

(i) All solutions of (17)-(18) are defined on $\left[t_{0}, \infty\right)$ for any initial condition $\left|x_{0}\right|<$ $\sqrt[a]{\frac{k_{1}}{k_{2}}} \delta$

(ii) moreover, the origin $x=0$ is uniformly stable and asymptotically stable.

Additionally, if Inequality (24) is satisfied for $\delta=\infty$ then $x=0$ is globally asymptotically stable.

Before giving the proof of Theorem 6 we compare it to the results obtained in 66. That reference considers systems of the form $\dot{x}=f(x, u)$ in which $u$ is the input, and $x\left(t_{0}\right)=x_{0}$ is the initial condition. Consider the set

$\Omega_{1}\left(t_{0}\right)=\left\{h \in C^{0}\left(\mathbb{R}, \mathbb{R}_{+}\right) / \exists \tau, \varepsilon, \bar{h}>0\right.$ satisfying $\int_{t-\tau}^{t} h(s) d s \geq \varepsilon$ and $\left.h(t) \leq \bar{h}, \forall t \geq t_{0}\right\}$.

If there exists a $C^{1}$ Lyapunov function $V$ such that $\frac{d}{d t} V(t, x(t), u(t)) \leq-h(t) \beta_{1}(|x(t)|)$ for almost all $t>t_{0}$ with $|x(t)| \geq \beta_{2}(|u(t)|)$ for some $h \in \Omega_{1}, \beta_{1} \in \mathscr{K}_{\infty} \cap C^{1}$ and some $\beta_{2} \in \mathscr{K}_{\infty}$, then the system is input-to-state stable (see Definition 1 and Theorem 5 in [6]).

Any continuous periodic function that is not identically zero belongs to the set $\Omega_{1}$. Additionally, there are nonperiodic functions that belong to $\Omega_{1}$ [6, p. 3].

The contribution of Theorem 6 is to provide sufficient conditions for the asymptotic stability of (17)-(18) for functions $h$ that belong to the set $\Omega_{2}$ which is larger than $\Omega_{1}$. 
Proposition 7. $\Omega_{1} \subset \Omega_{2}$ and $\Omega_{2} \backslash \Omega_{1}$ is nonempty.

Proof. To prove that $\Omega_{1} \subset \Omega_{2}$, assume that there exists a function $h: \mathbb{R} \rightarrow \mathbb{R}_{+}$such that $h \in \Omega_{1}$ and $h \notin \Omega_{2}$. Since $h \in \Omega_{1}$, then $h$ is continuous (and hence Lebesgue measurable) and there exist $\tau>0$ and there exists $\varepsilon>0$ such that $\int_{t-\tau}^{t} h(s) d s \geq \varepsilon$ for all $t \geq t_{0}$. On the other hand, since $h \notin \Omega_{2}$, then $\int_{t_{0}}^{\infty} h(s) d s \neq \infty$. Thus $\int_{t_{0}}^{\infty} h(s) d s=w \in \mathbb{R}_{+}$since $h \geq 0$. On the other hand, we conclude by the inequality $\int_{t-\tau}^{t} h(s) d s \geq \varepsilon$ for all $t \geq t_{0}$ that $\int_{t-\tau}^{t_{0}} h(s) d s+\int_{t_{0}}^{t} h(s) d s \geq \varepsilon$ for all $t \geq t_{0}$. Taking $t \rightarrow \infty$ leads to the contradiction $-w+w=0 \geq \varepsilon$. Therefore $\Omega_{1} \subset \Omega_{2}$.

To prove that $\Omega_{2} \backslash \Omega_{1}$ is nonempty, consider the continuous function $h: \mathbb{R} \rightarrow \mathbb{R}$ defined by $h(t)=\frac{1}{t+1}$ for all $t \geq t_{0}$ and $h(t)=\frac{1}{t_{0}+1}$ for all $t \leq t_{0}$. Then $\int_{t_{0}}^{\infty} h(s) d s=\infty$ so that $h \in \Omega_{2}$. Suppose that there exist $\tau>0$ and $\varepsilon>0$ such that for all $t \geq t_{0}+\tau$, $\int_{t-\tau}^{t} h(s) d s=\ln \left(\frac{t+1}{t-\tau+1}\right) \geq \varepsilon$. Taking $t \rightarrow \infty$ leads to a contradiction which shows that $h \in \Omega_{2} \backslash \Omega_{1}$.

Another example is $h: \mathbb{R} \rightarrow \mathbb{R}$ defined by $h(t)=t$ for all $t \in \mathbb{R}$ which is in $\Omega_{2} \backslash \Omega_{1}$.

We now present the proof of Theorem 6 .

Proof of Theorem 6. Let $x$ be a solution of (17)-(18) with maximal interval of existence $\left[t_{0}, \omega\right)$. Define $z:\left[t_{0}, \omega\right) \rightarrow \mathbb{R}_{+}$as $z(t)=V(t, x(t))$ for all $t \in\left[t_{0}, \omega\right)$. Since $x$ is absolutely continuous and $V$ is continuously differentiable, the function $z$ is absolutely continuous on each compact interval of $\left[t_{0}, \omega\right)$. Therefore, Condition (i) of Lemma 1 is satisfied. We get from (24) and (23) that

$$
\dot{z}(t)=\left.\frac{\partial V(t, \alpha)}{\partial t}\right|_{\alpha=x(t)}+\left.\frac{\partial V(t, \alpha)}{\partial \alpha}\right|_{\alpha=x(t)} \cdot f(t, x(t)) \leq 0 .
$$

for almost all $t \in\left(t_{0}, \omega\right)$ that satisfy $0<z(t)<k_{1} \delta^{a}$. Thus Condition (ii) of Lemma 1 is satisfied with $z_{1}=0$ and $z_{2}=k_{1} \delta^{a}$. It follows from Lemma 11 that if $z\left(t_{0}\right)<z_{2}=k_{1} \delta^{a}$ then $z(t) \leq z\left(t_{0}\right)$ for all $t \in\left[t_{0}, \omega\right)$. This fact combined with Inequalities (23) leads to

$$
|x(t)| \leq \sqrt[a]{\frac{k_{2}}{k_{1}}}\left|x_{0}\right|, \forall t \in\left[t_{0}, \omega\right) \text { whenever }\left|x_{0}\right|<\sqrt[a]{\frac{k_{1}}{k_{2}}} \delta .
$$

It also follows that $\omega=\infty$ whenever $\left|x_{0}\right|<\sqrt[a]{\frac{k_{1}}{k_{2}}} \delta$ [11, p. 71]. These facts mean that the equilibrium point $x=0$ is uniformly stable.

Assume that $\left|x_{0}\right|<\sqrt[a]{\frac{k_{1}}{k_{2}}} \delta$. Define $\chi:\left[t_{0}, \infty\right) \rightarrow \mathbb{R}_{+}$as $\chi(t)=\int_{t_{0}}^{t} h(\tau) d \tau$ for all $t \in\left[t_{0}, \infty\right)$. Since the function $h$ is nonnegative almost everywhere on $\left[t_{0}, \infty\right)$, the function 
$\chi$ is nondecreasing. Thus the fact that $h \in \Omega_{2}$ implies that there exists $T_{1} \in\left(t_{0}, \infty\right)$ such that

$$
\chi\left(T_{1}\right)>\max \left(\frac{1}{k_{1}^{\frac{b}{a}} \delta^{b}}, k_{2}^{\frac{b^{2}}{a^{2}}}\left(\frac{a}{b}\right)^{\frac{b}{a}}, \frac{1}{\delta^{b}}\left(\frac{k_{2}^{2}}{k_{1}}\right)^{\frac{b}{a}}\right)
$$

and hence

$$
\chi(t)>k_{2}^{\frac{b^{2}}{a^{2}}}\left(\frac{a}{b}\right)^{\frac{b}{a}}, \forall t \geq T_{1} .
$$

Let $\psi_{1}:\left[t_{0}, \infty\right) \rightarrow \mathbb{R}_{+}$be defined as $\psi_{1}(t)=k_{1} \delta^{a}$ for all $t \in\left[t_{0}, \infty\right)$. Since $\psi_{1}$ is a constant function, it is absolutely continuous on $\left[t_{0}, \omega\right)$. Define $\varphi_{1}:\left[t_{0}, \infty\right) \rightarrow \mathbb{R}_{+}$as

$$
\varphi_{1}(t)= \begin{cases}(\chi(t))^{-\frac{a}{b}} & t \in\left[T_{1}, \infty\right), \\ \left(\chi\left(T_{1}\right)\right)^{-\frac{a}{b}} & t \in\left[t_{0}, T_{1}\right)\end{cases}
$$

We have $\varphi_{1}(t) \leq\left(\chi\left(T_{1}\right)\right)^{-\frac{a}{b}}$ for all $t \geq t_{0}$ because the function $\chi$ is nondecreasing. Furthermore, it follows from 25 that $\chi\left(T_{1}\right)>1 / k_{1}^{\frac{b}{a}} \delta^{b}$. Therefore, we have

$$
\varphi_{1}(t)<\psi_{1}(t)=k_{1} \delta^{a}, \forall t \geq t_{0},
$$

and hence Inequality (9) in Proposition 2 is satisfied. The function $\varphi_{1}$ is continuous on $\left[t_{0}, \infty\right)$ and is continuously differentiable on the open set $\left(t_{0}, T_{1}\right) \cup\left(T_{1}, \infty\right)$ and thus it is absolutely continuous on each compact interval of $\left[t_{0}, \infty\right)$. Moreover, we have

$$
\dot{\varphi}_{1}(t)= \begin{cases}-\frac{a}{b}(\chi(t))^{-\frac{a}{b}-1} h(t) & t \in\left(T_{1}, \infty\right), \\ 0 & t \in\left(t_{0}, T_{1}\right) .\end{cases}
$$

Observe that $\dot{\varphi}_{1}(t) \leq 0$ for almost all $t>t_{0}$ because the function $h$ is nonnegative almost everywhere on $\left[t_{0}, \infty\right) \supset\left(T_{1}, \infty\right)$. Thus Inequalities (23) and (24) imply that

$$
\dot{z}(t) \leq 0=\min \left(\dot{\varphi}_{1}(t), \dot{\psi}_{1}(t)\right) \text { for almost all } t \in\left(t_{0}, T_{1}\right) \text { that satisfy } z(t)<k_{1} \delta^{a} .
$$

On the other hand, we obtain from $(23)$ and $(24)$ that

$$
\dot{z}(t) \leq-h(t)\left(\frac{z(t)}{k_{2}}\right)^{\frac{b}{a}} \text { for almost all } t>t_{0} \text { that satisfy } z(t)<\psi_{1}(t)=k_{1} \delta^{a} .
$$

It comes from $(26)$ that

$$
-h(t)\left(\frac{\varphi_{1}(t)}{k_{2}}\right)^{\frac{b}{a}} \leq \dot{\varphi}(t) \text { for almost all } t>T_{1},
$$


and thus we get from 30 that

$$
\begin{aligned}
\dot{z}(t) \leq \min \left(\dot{\varphi}_{1}(t), \dot{\psi}_{1}(t)\right) \leq & 0 \text { for almost all } t>T_{1} \\
& \text { that satisfy } \varphi_{1}(t)<z(t)<\psi_{1}(t)=k_{1} \delta^{a} .
\end{aligned}
$$

Combining Equations (31) and (29) it comes that Inequality (10) is satisfied. Therefore we get from (3) and (11) and Propositions 2 and 3 that

$$
\begin{aligned}
& z(t) \leq \varphi_{1}(t), \forall t \in\left[t_{0}, \infty\right) \text { whenever } z\left(t_{0}\right) \leq\left(\chi\left(T_{1}\right)\right)^{-\frac{a}{b}} \\
& z(t)<\psi_{1}(t)=k_{1} \delta^{a}, \forall t \in\left[t_{0}, \infty\right) \text { whenever }\left(\chi\left(T_{1}\right)\right)^{-\frac{a}{b}}<z\left(t_{0}\right)<k_{1} \delta^{a} .
\end{aligned}
$$

Recall that, owing to Inequality 25 , we have $\chi\left(T_{1}\right)>\frac{1}{\delta^{b}}\left(\frac{k_{2}^{2}}{k_{1}}\right)^{\frac{b}{a}}$. This fact along with Inequalities 23 imply that

$$
\begin{aligned}
& |x(t)| \leq \sqrt[a]{\frac{\varphi_{1}(t)}{k_{1}}}, \forall t \in\left[t_{0}, \infty\right), \text { whenever }\left|x_{0}\right| \leq \frac{1}{k_{2}^{\frac{1}{a}}\left(\chi\left(T_{1}\right)\right)^{\frac{1}{b}}}, \\
& |x(t)|<\delta, \forall t \in\left[t_{0}, \infty\right) \text { whenever } \frac{1}{k_{1}^{\frac{1}{a}}\left(\chi\left(T_{1}\right)\right)^{\frac{1}{b}}}<\left|x_{0}\right|<\sqrt[a]{\frac{k_{1}}{k_{2}}} \delta .
\end{aligned}
$$

Note that $\frac{1}{k_{1}^{1 / a}\left(\chi\left(T_{1}\right)\right)^{1 / b}}<\sqrt[a]{\frac{k_{1}}{k_{2}}} \delta$ because $\chi\left(T_{1}\right)>\frac{1}{\delta^{b}}\left(\frac{k_{2}^{2}}{k_{1}}\right)^{\frac{b}{a}}$. Observe that, owing to Equation (27), it comes that $\lim _{t \rightarrow \infty} \varphi_{1}(t)=0$ because $h \in \Omega_{2}$. Hence, it follows from 33 that $\lim _{t \rightarrow \infty} x(t)=0$ whenever $\left|x_{0}\right| \leq \frac{1}{k_{2}^{\frac{1}{a}}\left(\chi\left(T_{1}\right)\right)^{\frac{1}{b}}}$. Thus, the equilibrium $x=0$ is a locally attractive point although it is not a uniformly locally attractive point because $T_{1}$ depends on $t_{0}$.

To sum up, we have proved so far that $x=0$ is stable and locally attractive. This means that $x=0$ is asymptotically stable.

Finally, we need to prove that when Inequality (24) is satisfied for $\delta=\infty$ then $x=0$ is globally asymptotically stable. To this end, we first need to prove that $\omega=\infty$ for any initial condition $x_{0} \in \mathbb{R}^{m}$, where $\left[t_{0}, \omega\right)$ is the largest interval of existence of the Carathéodory solutions of $(17)-(18)$. This comes next.

We have from (24) that $\dot{z}(t) \leq 0$ for almost all $t \in\left(t_{0}, \omega\right)$. Thus all conditions of Lemma 1 are satisfied with $z_{1}=0$ and $z_{2}=z\left(t_{0}\right)+1$ and thus we have $z(t) \leq z\left(t_{0}\right)$ for all $t \in\left[t_{0}, \omega\right)$. Therefore $(23)$ implies that:

$$
|x(t)| \leq \sqrt[a]{\frac{k_{2}}{k_{1}}}\left|x_{0}\right|, \forall t \in\left[t_{0}, \omega\right) \text { for any } x_{0} \in \mathbb{R}^{m},
$$


and hence $\omega=\infty$ for any $x_{0} \in \mathbb{R}^{m}$.

The rest of the analysis is dedicated to proving that $x=0$ is globally asymptotically stable.

Note first that the fact that $h \in \Omega_{2}$ leads to the existence of $T_{2}>t_{0}$ such that

$$
\chi(t)>k_{2}^{\frac{b^{2}}{a^{2}}}\left(\frac{a}{b}\right)^{\frac{b}{a}}, \forall t \geq T_{2} .
$$

Let $\varphi_{2}:\left[t_{0}, \infty\right) \rightarrow \mathbb{R}_{+}$be defined as

$$
\varphi_{2}(t)= \begin{cases}(\chi(t))^{-\frac{a}{b}} & t \in\left[T_{2}, \infty\right), \\ \left(\chi\left(T_{2}\right)\right)^{-\frac{a}{b}} & t \in\left[t_{0}, T_{2}\right) .\end{cases}
$$

We have $\varphi_{2}(t) \leq\left(\chi\left(T_{2}\right)\right)^{-\frac{a}{b}}$ for all $t \geq t_{0}$ and $\lim _{t \rightarrow \infty} \varphi_{2}(t)=0$ because $\chi$ is nondecreasing and $h \in \Omega_{2}$. It comes from (34) that

$$
-h(t)\left(\frac{\varphi_{2}(t)}{k_{2}}\right)^{\frac{b}{a}} \leq \dot{\varphi}_{2}(t) \text { for almost all } t>T_{2} .
$$

Define a constant function $\psi_{2}:\left[t_{0}, \infty\right) \rightarrow \mathbb{R}_{+}$as

$$
\psi_{2}(t)=1+\left\|\varphi_{2}\right\|+z\left(t_{0}\right)=1+\left\|\varphi_{2}\right\|+V\left(t_{0}, x_{0}\right), \forall t \geq t_{0} .
$$

Observe that Inequality (9) is satisfied. On the other hand, Inequality (24) implies that

$$
\dot{z}(t) \leq 0=\min \left(\dot{\varphi}_{2}(t), \dot{\psi}_{2}(t)\right) \text { for almost all } t \in\left(t_{0}, T_{2}\right) .
$$

On the other hand, we obtain from (23) and 24) that

$$
\dot{z}(t) \leq-h(t)\left(\frac{z(t)}{k_{2}}\right)^{\frac{b}{a}} \text { for almost all } t>t_{0} .
$$

Hence 12), 13), and 14 are satisfied with $H(v)=v^{\frac{b}{a}}$ for all $v \geq 0$ and $q(t)=k_{2}^{-\frac{b}{a}} h(t)$ for all $t \geq t_{0}$. Moreover we get by (35) and (37) that

$$
\dot{z}(t) \leq \min \left(\dot{\varphi}_{2}(t), \dot{\psi}_{2}(t)\right) \leq 0 \text { for almost all } t>T_{2} \text { that satisfy } z(t)>\varphi_{2}(t) .
$$

Observe that (36) implies that Inequality (10) is satisfied. Therefore, all conditions of Corollary 4 are satisfied. This implies that for any $x_{0} \in \mathbb{R}^{m}$ we have $\lim _{t \rightarrow \infty} z(t)=0$. 
Therefore, we obtain from (23) that $\lim _{t \rightarrow \infty} x(t)=0$ for any initial condition $x_{0} \in \mathbb{R}^{m}$ and hence $x=0$ is globally attractive. Thus $x=0$ is globally asymptotically stable since it is stable.

We now apply Theorem 6 to the study of the asymptotic stability of a class of linear time-varying (LTV) systems. Unlike linear time-invariant systems, the stability of LTV systems cannot be linked with the location of the eigenvalues of their system matrix ([12]). For this reason, only special classes of LTV systems have been studied in the literature. In the following example we consider a second-order LTV system whose right-hand side may be discontinuous with respect to time. We provide sufficient conditions for the uniform stability and global asymptotic stability of the system.

Example 8. Consider the linear time-invariant system

$$
\begin{aligned}
\dot{x}(t) & =A x(t), \text { for almost all } t \in\left[t_{0}, \infty\right), \\
x\left(t_{0}\right) & =x_{0},
\end{aligned}
$$

where $n \in \mathbb{N} \backslash\{0\}, A$ is an $n \times n$ real stable matrix, that is every eigenvalue of $A$ has a strictly negative real part, $t_{0} \in \mathbb{R}, x_{0} \in \mathbb{R}^{n}$, and state $x(t) \in \mathbb{R}^{n}$. Let $P$ be the real symmetric positive-definite $n \times n$ matrix that satisfies $P A+A^{T} P=I_{n}$ where $I_{n}$ is the $n \times n$ identity matrix. Consider the Lyapunov function $V(x)=x^{T} P x$, then $V$ satisfies (23) and (24) with $a=b=2$ and $h(t)=1$ for all $t \geq t_{0}, k_{1}$ and $k_{2}$ being the smallest and largest singular values of $A$. Since we can take $\delta=\infty$ it follows from Theorem 6 (ii) that the origin is globally asymptotically stable.

Example 8 shows that when Theorem 6 is applied to linear time-invariant systems, the obtained stability result is not conservative.

Example 9. Consider the linear time-varying system

$$
\begin{aligned}
\dot{x}(t) & =A(t) x(t), \text { for almost all } t \in\left[t_{0}, \infty\right), \\
x\left(t_{0}\right) & =x_{0},
\end{aligned}
$$

where $t_{0} \in \mathbb{R}, x_{0} \in \mathbb{R}^{2}$, and state $x(t) \in \mathbb{R}^{2}$. The matrix $A$ is defined by

$$
A(t)=\left[\begin{array}{ll}
c_{1}(t) & c_{2}(t) \\
c_{3}(t) & c_{4}(t)
\end{array}\right]
$$

for some measurable functions $c_{i}:\left[t_{0}, \infty\right) \rightarrow \mathbb{R}, i=1,2,3,4$. Assume that

$$
c_{1}(t)<0 \text { and } c_{4}(t)<0 \text { for almost all } t \in\left[t_{0}, \infty\right),
$$




$$
\begin{gathered}
0 \leq c_{2}(t)+c_{3}(t)<2 \min \left(\left|c_{1}(t)\right|,\left|c_{4}(t)\right|\right) \text { for almost all } \\
\lim _{t \rightarrow \infty} \int_{t_{0}}^{t} c_{1}(\tau) d \tau=\lim _{t \rightarrow \infty} \int_{t_{0}}^{t} c_{4}(\tau) d \tau=-\infty \\
\lim _{t \rightarrow \infty} \int_{t_{0}}^{t}\left(c_{2}(\tau)+c_{3}(\tau)\right) d \tau<\infty .
\end{gathered}
$$

Since the matrix function $A(t)$ is measurable, the system (39) satisfies all assumptions of [3, Theorem 3]. This implies that a unique absolutely continuous solution of (39) exists on $\left[t_{0}, \infty\right)$.

Observe that $x=0$ is an equilibrium point for (39).

Define $h:\left[t_{0}, \infty\right) \rightarrow \mathbb{R}_{+}$for almost all $t \geq t_{0}$ as

$$
h(t)=-\max \left(c_{1}(t)+\frac{c_{2}(t)+c_{3}(t)}{2}, c_{4}(t)+\frac{c_{2}(t)+c_{3}(t)}{2}\right) .
$$

Owing to 40-43 it comes that $h$ is measurable, nonnegative, and $\lim _{t \rightarrow \infty} \int_{t_{0}}^{t} h(\tau) d \tau=$ $\infty$.

Consider the continuously differentiable quadratic Lyapunov function candidate $V: \mathbb{R}^{2} \rightarrow$ $\mathbb{R}_{+}$defined by

$$
V(\alpha)=\frac{1}{2}|\alpha|^{2}, \forall \alpha \in \mathbb{R}^{2} .
$$

Then Inequality (23) is satisfied with $k_{1}=k_{2}=1 / 2$ and $a=2$. We get for almost all $t \in\left[t_{0}, \infty\right)$ that

$$
\begin{aligned}
\left.\frac{d V(\alpha)}{d \alpha}\right|_{\alpha=x(t)=\left(x_{1}(t), x_{2}(t)\right)} \cdot f(t, x(t)) & =\left[\begin{array}{ll}
x_{1}(t) & x_{2}(t)
\end{array}\right] A(t)\left[\begin{array}{l}
x_{1}(t) \\
x_{2}(t)
\end{array}\right] \\
& =c_{1}(t) x_{1}^{2}+c_{4}(t) x_{2}^{2}+\left(c_{2}(t)+c_{3}(t)\right) x_{1} x_{2} \\
& \leq\left(c_{1}(t)+\frac{c_{2}(t)+c_{3}(t)}{2}\right) x_{1}^{2} \\
& +\left(c_{4}(t)+\frac{c_{2}(t)+c_{3}(t)}{2}\right) x_{2}^{2} \\
& \leq-h(t)|x(t)|^{2},
\end{aligned}
$$


Thus (24) is satisfied globally with $b=2$ and $\delta=\infty$. Thus, it follows from Theorem 6 that $x=0$ is uniformly stable and is globally asymptotically stable.

Note that the special case $c_{2}(t)=-c_{3}(t)$ makes Conditions (41) and (43) trivially satisfied.

\section{Asymptotic stability of perturbed linear systems}

In this section we study the stability of the following class of perturbed linear systems:

$$
\begin{aligned}
\dot{x}(t) & =A x(t)+g(t, x(t)), \\
x\left(t_{0}\right) & =x_{0},
\end{aligned}
$$

where $t \geq t_{0}, A$ is a $m \times m$ matrix, state $x(t) \in \mathbb{R}^{m}$, and a function $g:\left[t_{0}, \infty\right) \times \mathbb{R}^{m} \rightarrow \mathbb{R}^{m}$. In this section, we assume that

$\left(\mathrm{B}_{1}\right)$ the function $g(t, x)$ is locally essentially bounded on $\mathbb{R}_{+} \times \mathbb{R}^{m}$,

$\left(\mathrm{B}_{2}\right)$ for each $x \in \mathbb{R}^{m}$, the function $t \mapsto g(t, x)$ is measurable,

$\left(\mathrm{B}_{3}\right)$ for almost all $t \in \mathbb{R}_{+}$, the function $x \mapsto g(t, x)$ is continuous,

$\left(\mathrm{B}_{4}\right)$ and for all $t \geq 0, g(t, 0)=0$.

Assumptions $\left(\mathrm{B}_{1}\right)-\left(\mathrm{B}_{3}\right)$ imply that the right-hand side of $(44)$ satisfies Assumptions $\left(\mathrm{A}_{1}\right)$ $\left(\mathrm{A}_{3}\right)$. This implies that a local Carathéodory solution for (44)-(45) exists and is defined on an interval of the form $\left[t_{0}, \omega\right), t_{0}<\omega \leq \infty$. Assumption $\left(\mathrm{B}_{4}\right)$ means that $x=0$ is an equilibrium point for 44).

In the following theorem, we introduce sufficient conditions for the asymptotic stability of the system (44)-(45). The main novelty of the result is that the perturbation term $g(t, \alpha)$ may be unbounded with respect to time.

Theorem 10. Suppose that the following Conditions 1-4 hold.

1. The matrix $A$ is stable.

2. There exist $d>1, \delta \in(0, \infty]$ and a function $G \in C^{1}\left(\left[t_{0}, \infty\right), \mathbb{R}_{+}\right)$such that

$$
|g(t, \alpha)| \leq G(t)|\alpha|^{d}, \forall t \geq t_{0}, \text { and } \forall \alpha \in \mathbb{R}^{m} \text { with }|\alpha|<\delta .
$$

3. The function $G$ is nondecreasing on $\left[t_{0}, \infty\right)$ with $\lim _{t \rightarrow \infty} G(t)=\infty$.

4. For some instant $t_{1} \geq t_{0}$ that satisfies $G(t)>0$ for all $t \geq t_{1}$, the function $\Phi$ : $\left[t_{1}, \infty\right) \rightarrow \mathbb{R}_{+}$which is defined as $\Phi(t)=\frac{\dot{G}(t)}{G(t)}$, for all $t \geq t_{1}$, is nonincreasing with $\lim _{t \rightarrow \infty} \Phi(t)=0$. 
Then

(i) there exists $r>0$ such that if $\left|x_{0}\right| \leq r$, then each solution $x(t)$ of (44)-(45) is continuable on $\left[t_{0}, \infty\right)$,

(ii) the origin $x=0$ is asymptotically stable; in particular we have $\lim _{t \rightarrow \infty} x(t)=0$ whenever $\left|x_{0}\right| \leq r$. Moreover, if $g$ is continuous then $\lim _{t \rightarrow \infty} \dot{x}(t)=0$ whenever $\left|x_{0}\right| \leq r$.

Proof. Since $A$ is stable there exists a symmetric $m \times m$ matrix $P>0$ that satisfies

$$
P A+A^{T} P=-I
$$

where $I$ is the identity $m \times m$ matrix [4, p.136]. Consider the quadratic Lyapunov function candidate $V \in C^{1}\left(\mathbb{R}^{m}, \mathbb{R}\right)$ with $V(\alpha)=\alpha^{T} P \alpha$ for all $\alpha \in \mathbb{R}^{m}$. Then [4, p. 155]

$$
\lambda_{\min }|\alpha|^{2} \leq V(\alpha) \leq \lambda_{\max }|\alpha|^{2},
$$

where $\lambda_{\min }>0$ and $\lambda_{\max }>0$ are respectively the minimum and the maximum eigenvalues of the matrix $P$. Thus, we get

$$
\begin{gathered}
\frac{d V(\alpha)}{d \alpha} \cdot A \alpha=-|\alpha|^{2}, \forall \alpha \in \mathbb{R}^{m}, \\
\left|\frac{d V(\alpha)}{d \alpha}\right|=2|P \alpha| \leq 2|P|_{2}|\alpha|=2 \lambda_{\max }|\alpha|, \forall \alpha \in \mathbb{R}^{m},
\end{gathered}
$$

where $|P|_{2}$ is the induced 2-norm for the matrix $P$. Therefore

$$
\frac{d V(\alpha)}{d \alpha} \cdot(A \alpha+g(t, \alpha)) \leq-|\alpha|^{2}+2 \lambda_{\max }|\alpha||g(t, \alpha)|, \forall t \in\left[t_{0}, \infty\right), \forall \alpha \in \mathbb{R}^{m}
$$

Let $x(t)$ be a solution of $(44)-(45)$ with maximal interval of existence $\left[t_{0}, \omega\right)$. Define $z:\left[t_{0}, \omega\right) \rightarrow \mathbb{R}_{+}$as $z(t)=V(x(t))$ for all $t \in\left[t_{0}, \omega\right)$. Since $x$ absolutely continuous and $V$ is continuously differentiable, the function $z$ is absolutely continuous on each compact interval of $\left[t_{0}, \omega\right)$. We obtain from (46) and that

$$
\begin{aligned}
\dot{z}(t) \leq-|x(t)|^{2}+2 \lambda_{\max } G(t)|x(t)|^{d+1} & \text { for almost all } t \in\left(t_{0}, \omega\right) \\
& \text { that satisfy }|x(t)|<\delta .
\end{aligned}
$$

On the other hand, Condition 4 and the fact that $G$ is nondecreasing imply that there exists $t_{2}>t_{1}$ such that

$$
\Phi(t)=\frac{\dot{G}(t)}{G(t)} \leq \frac{d-1}{8 \lambda_{\max }}, \text { for all } t \geq t_{2}
$$


and

$$
\frac{1}{\sqrt[d-1]{4 \lambda_{\max } G\left(t_{2}\right)}}<\delta
$$

Let $\psi, \varphi:\left[t_{0}, \omega\right) \rightarrow \mathbb{R}_{+}$be defined as

$$
\psi(t)= \begin{cases}\lambda_{\min }\left(\frac{1}{4 \lambda_{\max } G\left(t_{2}\right)}\right)^{\frac{2}{d-1}} & \text { if } t_{0} \leq t<\min \left(t_{2}, \omega\right) \\ \lambda_{\min }\left(\frac{1}{4 \lambda_{\max } G(t)}\right)^{\frac{2}{d-1}} & \text { if } t_{2} \leq t<\omega \text { and } t_{2}<\omega\end{cases}
$$

and $\varphi(t)=\frac{1}{2} \psi(t)$ for all $t \in\left[t_{0}, \omega\right)$. The functions $\psi$ and $\varphi$ are absolutely continuous on each compact interval of $\left[t_{0}, \omega\right)$ because the function $G:\left[t_{0}, \infty\right) \rightarrow \mathbb{R}_{+}$is continuously differentiable. Thus Condition (i) of Proposition 2 is satisfied. From (52) and the definitions of $\varphi$ and $\psi$, we conclude that

$$
\varphi(t)<\psi(t)<\lambda_{\min } \delta^{2}, \forall t \in\left[t_{0}, \omega\right)
$$

and hence Inequality (9) is satisfied.

Since the function $G$ is nondecreasing on $\left[t_{0}, \infty\right)$, we have

$$
\dot{\psi}(t) \leq \dot{\varphi}(t) \leq 0, \text { for almost all } t \in\left(t_{0}, \omega\right) .
$$

Moreover, it comes from (52) and (50) that if $t_{2}<\omega$, then

$$
\dot{z}(t) \leq-\frac{1}{2}|x(t)|^{2}, \text { for almost all } t \in\left(t_{2}, \omega\right) \text { that satisfy }|x(t)|<\frac{1}{\sqrt[d-1]{4 \lambda_{\max } G(t)}} .
$$

Claim 11. We have

$$
\begin{gathered}
\dot{z}(t) \leq-\frac{1}{2}|x(t)|^{2}, \text { for almost all } t \in\left(t_{0}, \min \left(t_{2}, \omega\right)\right) \text { that satisfy } \\
\qquad|x(t)|<\frac{1}{\sqrt[d-1]{4 \lambda_{\max } G\left(t_{2}\right)}} .
\end{gathered}
$$

Proof. Let $S=\left\{\tau \in\left(t_{0}, \min \left(t_{2}, \omega\right)\right) / G(t)=0\right.$, for all $\left.t \in\left(t_{0}, \tau\right)\right\}$.

If $S=\emptyset$, then the fact that $G \in C^{1}\left(\left[t_{0}, \infty\right), \mathbb{R}_{+}\right)$is nondecreasing implies that $G(t)>0$ for all $t \in\left(t_{0}, \min \left(t_{2}, \omega\right)\right)$. Thus

$$
\frac{1}{\sqrt[d-1]{4 \lambda_{\max } G\left(t_{2}\right)}} \leq \frac{1}{\sqrt[d-1]{4 \lambda_{\max } G(t)}}, \forall t \in\left(t_{0}, \min \left(t_{2}, \omega\right)\right)
$$


and hence (50) and (52) imply that (56) is satisfied.

If $S=\left(t_{0}, \min \left(t_{2}, \omega\right)\right)$, then $G(t)=0$ for all $t \in\left(t_{0}, \min \left(t_{2}, \omega\right)\right)$ and thus (56) is satisfied because of (50) and (52).

If $S \neq \emptyset$ and $S \neq\left(t_{0}, \min \left(t_{2}, \omega\right)\right)$, then the continuity of $G$ implies that $T_{3}=\sup S \in$ $\left(t_{0}, \min \left(t_{2}, \omega\right)\right)$. Thus $G(t)=0$ for all $t \in\left(t_{0}, T_{3}\right)$ and $G(t)>0$ for all $t \in\left(T_{3}, \min \left(t_{2}, \omega\right)\right)$. By considering the intervals $\left(t_{0}, T_{3}\right)$ and $\left(T_{3}, \min \left(t_{2}, \omega\right)\right)$ it can be verified that (56) is satisfied.

We have from (48), (55) and (56) that

$$
\dot{z}(t) \leq-\frac{1}{2 \lambda_{\max }} z(t) \text {, for almost all } t \in\left(t_{0}, \omega\right) \text { that satisfy } z(t)<\psi(t) .
$$

Claim 12. If $t_{2}<\omega$, then

$$
\dot{z}(t) \leq \min (\dot{\varphi}(t), \dot{\psi}(t)) \text { for almost all } t \in\left(t_{2}, \omega\right) \text { that satisfy } \varphi(t)<z(t)<\psi(t) \text {. }
$$

Proof. We deduce from (51) that

$$
-\frac{1}{4 \lambda_{\max }} \leq \frac{2}{(d-1)}\left(\frac{1}{4 \lambda_{\max } G(t)}\right)^{-1} \frac{d}{d t}\left(\frac{1}{4 \lambda_{\max } G(t)}\right) \text { for almost all } t>t_{2} .
$$

By multiplying both sides by $\lambda_{\min }\left(\frac{1}{4 \lambda_{\max } G(t)}\right)^{\frac{2}{d-1}}$, we get

$$
-\frac{1}{2 \lambda_{\max }} \varphi(t) \leq \dot{\psi}(t) \text { for almost all } t>t_{2}
$$

On the other hand, one has $\dot{\psi}(t)=\min (\dot{\varphi}(t), \dot{\psi}(t))$ for almost all $t \in\left(t_{2}, \omega\right)$ because of (54). Therefore, Inequalities (57) and (58) complete the proof.

Claim 12 and the fact that $\dot{\psi}(t)=\dot{\varphi}(t)=0$, for almost all $t \in\left(t_{0}, \min \left(t_{2}, \omega\right)\right)$ imply that

$$
\dot{z}(t) \leq \min (\dot{\varphi}(t), \dot{\psi}(t)) \text { for almost all } t \in\left(t_{0}, \omega\right) \text { that satisfy } \varphi(t)<z(t)<\psi(t) .
$$

Therefore, Inequality (10) is satisfied. Equation (3) of Proposition 2 gives

$$
z(t) \leq \varphi(t), \forall t \in\left[t_{0}, \omega\right) \text { whenever } z\left(t_{0}\right) \leq \varphi\left(t_{0}\right)=\frac{\lambda_{\min }}{2\left(4 \lambda_{\max } G\left(t_{2}\right)\right)^{\frac{2}{d-1}}},
$$


which together with 48 lead to

$$
|x(t)| \leq \sqrt{\frac{\varphi(t)}{\lambda_{\min }}}, \forall t \in\left[t_{0}, \omega\right) \text { whenever }\left|x_{0}\right| \leq r
$$

where $r=\frac{\sqrt{\frac{\lambda_{\min }}{2}}}{\sqrt[d-1]{4\left(\lambda_{\max }\right)^{\frac{d+1}{2}} G\left(t_{2}\right)}}$. Note that 53 implies that $\omega=\infty$ whenever $\left|x_{0}\right| \leq r$.

In the rest of the proof, we assume that $\left|x_{0}\right| \leq r$.

Since $\omega=\infty$ and $\lim _{t \rightarrow \infty} G(t)=\infty$ we have $\lim _{t \rightarrow \infty} \varphi(t)=\lim _{t \rightarrow \infty} \psi(t)=0$. Thus we get from (59) that

$$
\lim _{t \rightarrow \infty} x(t)=0 \text { whenever }\left|x_{0}\right| \leq r .
$$

Hence $x=0$ is a locally attractive equilibrium point for (44). Note that $x=0$ is not uniformly locally attractive because $r$ depends on $t_{0}$.

Since the function $\varphi$ is nonincreasing, Inequality (4) is satisfied because of (57). Therefore, $z(t) \leq z\left(t_{0}\right)$ for all $t \geq t_{0}$, whenever $z\left(t_{0}\right) \leq \varphi\left(t_{0}\right)$ (see (5)). Hence we deduce from (48) that

$$
|x(t)| \leq \sqrt{\frac{\lambda_{\max }}{\lambda_{\min }}}\left|x_{0}\right|, \forall t \geq t_{0}, \text { whenever }\left|x_{0}\right| \leq r .
$$

Thus, the equilibrium $x=0$ is stable. This implies that this equilibrium is asymptotically stable because it is locally attractive.

Finally, we deduce from $(53)$ and $(59)$ that

$$
|x(t)|<\delta, \forall t \geq t_{0} \text { whenever }\left|x_{0}\right| \leq r .
$$

Therefore, (44) and (46) imply that

$$
|\dot{x}(t)| \leq|A|_{2}|x(t)|+G(t)|x(t)|^{d}, \text { for almost all } t>t_{0},
$$

where $|A|_{2}$ is the induced 2-norm of the matrix $A$. Hence, we obtain from (59) and the definition of the function $\varphi$ that

$$
|\dot{x}(t)| \leq|A|_{2}|x(t)|+G(t)\left(\frac{1}{\sqrt{2}\left(4 \lambda_{\max } G(t)\right)^{\frac{1}{d-1}}}\right)^{d} \text {, for almost all } t>t_{2},
$$

so that

$$
|\dot{x}(t)| \leq|A|_{2}|x(t)|+\frac{c}{(G(t))^{\frac{d}{d-1}}} \text {, for almost all } t>t_{2},
$$


where $c=\frac{1}{2^{\frac{d}{2}}\left(4 \lambda_{\max }\right)^{\frac{d}{d-1}}}$. If $g$ is continuous, then Inequality 62 holds for all $t>t_{2}$. Thus, 60) and the fact that $\lim _{t \rightarrow \infty} G(t)=\infty$ lead to

$$
\lim _{t \rightarrow \infty} \dot{x}(t)=0, \text { whenever }\left|x_{0}\right| \leq r .
$$

Example 13. Consider the second-order system

$$
\begin{aligned}
& \dot{x}_{1}(t)=x_{2}(t), \\
& \dot{x}_{2}(t)=-4 x_{1}(t)-2 x_{2}(t)-t^{4} x_{2}^{3}(t),
\end{aligned}
$$

where $t \geq t_{0} \geq 0, x_{1}$ and $x_{2}$ take values in $\mathbb{R}$. The system (63)- 64 can be rewritten as (44) where $x(t)=\left(x_{1}(t), x_{2}(t)\right), t \geq t_{0}$,

$$
A=\left[\begin{array}{cc}
0 & 1 \\
-4 & -2
\end{array}\right]
$$

and

$$
g(t, \alpha)=\left(0,-t^{4} \alpha_{2}^{3}\right), \forall t \geq t_{0}, \forall \alpha=\left(\alpha_{1}, \alpha_{2}\right) \in \mathbb{R}^{2} .
$$

The right-hand sides of (63)-(64) are continuous and satisfies a local Lipschitz condition with respect to $x .{ }^{2}$ Hence (63)-(64) has a unique continuously differentiable solution $x(t)$ that is defined on an interval of the form $\left[t_{0}, \omega\right), t_{0}<\omega \leq \infty$ [11, pp. 70]. Define $z:\left[t_{0}, \omega\right) \rightarrow \mathbb{R}_{+}$as $z(t)=x^{2}(t)$ for all $t \in\left[t_{0}, \omega\right)$. Since $x$ is continuously differentiable, the function $z$ is absolutely continuous on each compact interval of $\left[t_{0}, \omega\right)$.

The eigenvalues of the matrix $A$ are $-1+i \sqrt{3}$ and $-1-i \sqrt{3}$ where $i=\sqrt{-1}$. This implies that $A$ is stable and hence Condition 1 of Theorem 10 is satisfied.

On the other hand, we deduce from (65) that

$$
|g(t, \alpha)|=t^{4} \alpha_{2}^{3} \leq t^{4}\left(\sqrt{\alpha_{1}^{2}+\alpha_{2}^{2}}\right)^{3}=t^{4}|\alpha|^{3}, \forall t \geq t_{0}, \forall \alpha=\left(\alpha_{1}, \alpha_{2}\right) \in \mathbb{R}^{2},
$$

which implies that (46) is satisfied with $d=3, \delta=\infty$ and $G(t)=t^{4}$ for all $t \geq t_{0}$. This means that Condition 2 of Theorem 10 is satisfied. Condition 3 of Theorem 10 is also satisfied.

\footnotetext{
${ }^{2}$ We say that a function $f: \mathbb{R} \times \mathbb{R}^{m} \rightarrow \mathbb{R}$ satisfies a local Lipschitz condition with respect to $x$ if for any compact set $K \subset \mathbb{R} \times \mathbb{R}^{m}$, there exists a constant $L=L(K)>0$ such that for any $\left(t, \alpha_{1}\right),\left(t, \alpha_{2}\right) \in K$, we have $\left|f\left(t, \alpha_{2}\right)-f\left(t, \alpha_{1}\right)\right|<L\left|\alpha_{1}-\alpha_{2}\right|$ [11, pp. 66].
} 
Let $t_{1}>t_{0} \geq 0$, then $G(t)=t^{4}>0$ for all $t \geq t_{1}$. The function $\Phi:\left[t_{1}, \infty\right) \rightarrow \mathbb{R}_{+}$which is defined as $\Phi(t)=\frac{\dot{G}(t)}{G(t)}=\frac{4}{t}$, for all $t \geq t_{1}>0$ is strictly decreasing with $\lim _{t \rightarrow \infty} \Phi(t)=0$. Hence Condition 4 of Theorem 10 is satisfied. Thus, using Theorem 10 it comes that

1. There exists $r>0$ such that if $\left|x_{0}\right| \leq r$ then $\omega=\infty$.

2. The origin $x=0$ is asymptotically stable; in particular we have $\lim _{t \rightarrow \infty} x(t)=0$ whenever $\left|x_{0}\right| \leq r$. Moreover, $\lim _{t \rightarrow \infty} \dot{x}(t)=0$ whenever $\left|x_{0}\right| \leq r$.

Simulations: Take $t_{0}=0$ and $x_{0}=(0.02,-0.02)$. It can be observed in Fig. 1 that $\lim _{t \rightarrow \infty} x(t)=0$. Fig. 2 shows that $\lim _{t \rightarrow \infty} \dot{x}(t)=0$.

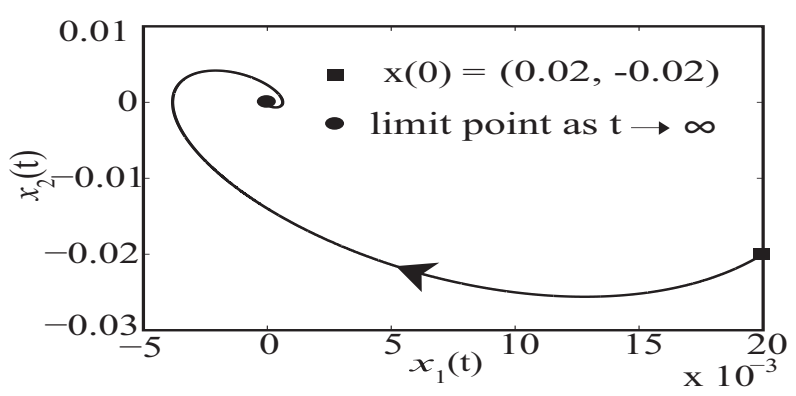

Figure 1: $x_{2}(t)$ versus $x_{1}(t)$ for system 63$)-64$.

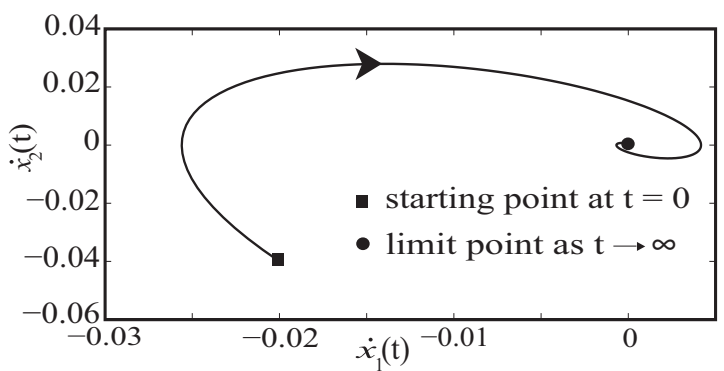

Figure 2: $\dot{x}_{2}(t)$ versus $\dot{x}_{1}(t)$ for system 63$)-64$.

\section{Conclusions}

This paper dealt with the asymptotic stability of nonlinear time-varying systems. The study is cast within the following framework: given an unknown absolutely continuous 
function $z \geq 0$, provide sufficient conditions on $z$ that allow (1) the knowledge of an upper bound on $z$ and (2) show that $z$ vanishes at infinity. This framework is shown to be useful for the analysis of the asymptotic stability of some classes of linear and nonlinear time-varying systems.

\section{Acknowledgment}

Supported by the Spanish Ministry of Economy, Industry and Competitiveness through grant DPI2016-77407-P (AEI/FEDER, UE).

[1] Aeyels, D. \& Peuteman, J. (1998) A new asymptotic stability criterion for nonlinear time-variant differential equations, IEEE Transactions on Automatic Control, 43, 968-971.

[2] Bacciotti, A. \& Rosier, L. (2005) Liapunov Functions and Stability in Control Theory. Springer.

[3] Filippov, A.F. (1988) Differential Equations with Discontinuous Right-Hand Sides. Kluwer.

[4] Khalil, H.K. (2002) Nonlinear Systems. 3rd ed., Prentice Hall, Upper Saddle River, New Jersey, ISBN 0130673897.

[5] Loría, A., Panteley, E., Popović, D. \& Teel, A.R. (2005) A nested Matrosov theorem and persistency of excitation for uniform convergence in stable nonautonomous systems, IEEE Transactions on Automatic Control, 50, 183-198.

[6] Malisoff, M. \& Mazenc, F. (2005) Further remarks on strict input-to-state stable Lyapunov functions for time-varying systems, Automatica, 41, 1973-1978.

[7] Mazenc, F. (2003) Strict Lyapunov functions for time-varying systems, Automatica, 39, 349-353.

[8] Mu, X. \& Cheng, D. (2005) On the stability and stabilization of time-varying nonlinear control systems, Asian Journal of Control, 7, 244-255.

[9] Naser, M.F.M. \& Ikhouane, F. (2013) Consistency of the Duhem model with hysteresis, Mathematical Problems in Engineering, 2013, ID 586130.

[10] Scarciotti, G. \& Praly, L. \& Astolfi, A. (2016) Invariance-like theorems and weak convergence properties, IEEE Transactions on Automatic Control, 61, 648-661. 
[11] Schmitt, K. \& Thompson, R. (1988) Nonlinear Analysis and Differential Equations: An Introduction, Lecture Notes. University of Utah, Department of Mathematics.

[12] Wu, M.Y. (1974) A note on stability of linear time-varying systems, IEEE Transactions on Automatic Control, 19, 162.

[13] Yen, E.H. \& Van Der VaArt, H.R. (1966) On measurable functions, continuous functions and some related concepts, The American Mathematical Monthly, 73, 991993. 